\title{
Transient orientational order and transient positional order in the sponge $\left(L_{3}\right)$ phase
}

\author{
Helmut R. Brand ${ }^{1}$ and Harald Pleiner ${ }^{2}$ \\ 1. Theoretische Physik III, Universität Bayreuth, D-95440 Bayreuth, Germany \\ 2. Max-Planck-Institut für Polymerforschung, Postfach 3148, D-55021 Mainz, Germany \\ Physica A312, 79 (2002)

\begin{abstract}
The question which macroscopic physical quantities are suitable to describe the optically isotropic sponge $\left(L_{3}\right)$ phase, which occurs in many lyotropic systems, is still a matter of debate. In this paper we analyze recent experimental results, which probed the response of sponge phases to jumps in applied pressure, temperature and electric field. Data obtained from dynamic light scattering are also incorporated into the discussion. As a result it emerges that the experimental data are compatible with the simultaneous occurrence of transient positional and transient orientational order. Additional experiments to further test the results of our analysis are suggested.
\end{abstract}

PACS: 61.30.St, 82.70.Uv, 05.70.Ln,

\section{Introduction}

The sponge [1] or $L_{3}$ [2] phase is observed in many lyotropic systems [3-10], typically between a lamellar $L_{\alpha}$ phase and an isotropic liquid phase. The sponge phase is optically isotropic, but shows strong linear flow birefringence [4]. Freeze fracture electron microscopy has provided quite detailed structural informations [11]: it is a bicontinuous phase for which the two continuous subphases are separated by bilayers. Locally isomorphic to a $L_{\alpha}$ phase, it can be transformed into the latter one by applying external shear $[12,13]$.

The question how one should describe the differences between an isotropic liquid phase and the $L_{3}$ phase attracted considerable attention. For the most part scalar order parameters to describe the static properties of the sponge phase were considered [1,14-16]. In 1991 [17] we analyzed the question of the order in the framework of a macroscopic 
description. Based on the observation of strong, linear flow birefringence [4] in the optically isotropic phase, we investigated which types of order would be compatible with these observations. It turned out that permanent orientational order as well as permanent positional order could be ruled out [17]. In addition it was shown that a scalar order parameter was also not a candidate to explain the observed flow birefringence. We pointed out that the macroscopic candidates remaining were transient positional and transient orientational order and we suggested concrete experiments how to test the predictions made [17].

Remarkably the number of dynamic experimental studies of the macroscopic properties of the sponge phase has remained rather small. Several groups reported their results of electric birefringence measurements on a number of systems varying the surfactant volume fraction and the chain length of the alkyl-chain [18-20]. They all found that their experimental results could be described by a single relaxation time. There was also an investigation employing $T$ jump measurements reporting three time constants [21], a rather surprising result.

Last year Strey's group presented the results of extensive dynamic measurements using a number of different techniques [22]. It is the goal of this manuscript to analyze the experimental results of dynamic measurements on the sponge phase in the framework of macroscopic dynamics and to compare them critically with the expectations from our early model [17].

\section{Interpretation of recent experimental results on the sponge phase}

In [22] several techniques have been used to investigate the macroscopic dynamic properties of the sponge phase in the ternary $\mathrm{H}_{2} \mathrm{O}-\mathrm{C}_{10} E_{4}-n-$ decanol $\left(C_{10} E_{4}\right)$ system.

A step change in temperature was followed up upon by light scattering. As a result the authors [22] found a relaxation with one relaxation time, $\tau_{T}$, which did not depend on the wave vector $\mathbf{q}$ with $q=|\mathbf{q}|$, that is $\tau_{T} \sim q^{0}$. The relaxation time $\tau_{T}$ was found to be in the range $1 \mathrm{~ms} . . .1 \mathrm{~s}$.

The second type of experiments described in ref. [22] was a jump in pressure and the associated dynamic detection method was the measurement of electric conductivity as a function of time. Again it was found that there was only one relaxation time $\tau_{P}$. As already for the case of the relaxation following a temperature jump, the relaxation time $\tau_{P}$ following a pressure jump was found to be in the range $1 \mathrm{~ms} . . .1 \mathrm{~s}$. And - as noted by the authors of ref. [22] - the two time scales were the same. From this observation it follows that the relaxation was independent of the wave vector and thus that the relaxation time $\tau_{P}$ following a pressure jump behaves as $\tau_{P} \sim q^{0}$. It seems also important to mention in this connection that the temperature dependence as well as the dependence on surfactant volume fraction $\Phi$ were the same for the relaxation times $\tau_{T}$ and $\tau_{P}$, where the temperature dependence showed an Arrhenian behavior. In addition, the $\Phi$-dependence of $\tau_{P}$ and $\tau_{T}$ was the strongest among all the processes studied.

The third technique used [22] was a jump in the electric field $\mathbf{E}$ followed along by detection of the electric current and the electric birefringence. The time scales for the associated relaxation time $\tau_{E}$ were in the range $\tau_{E} \sim 2 \ldots 200 \mu \mathrm{s}$ and thus much shorter than the relaxation times measured after a $p$-jump and a $T$-jump. The birefringence 
signal measured was $\sim E^{2}$ as expected.

The last technique used was dynamic light scattering. It was found [22] that the time scale associated with dynamic light scattering, $\tau_{D L S}$, depends quadratically on the wave vector: $\tau_{D L S}^{-1} \sim q^{2}$. The $\Phi$-dependence was also found to be much weaker that that for the relaxation times measured using the other three techniques.

To sum up, in the most extensive experimental investigations on the dynamic properties of the sponge phase reported so far [22], two types of relaxation and one diffusive mode were detected using four techniques (jumps in pressure, temperature and electric field as well as dynamic light scattering).

\section{Transient positional and orientational order: an analysis of recent experimental results on the sponge phase}

Now we are prepared to analyze the description of the experimental results given in the last section. To do this we use the approach of macroscopic dynamics, which we have used before to find out with which type of order early experiments on flow birefringence were compatible with [17]. At that time it turned out that only transient orientational order and transient positional order were compatible with the flow birefringence data, while permanent orientational order and permanent positional order as well as scalar variables were ruled out as candidates to characterize the order in the sponge phase [17].

It is straightforward to interpret the results of the dynamic light scattering data macroscopically. The observed diffusive mode corresponds to a dispersion relation of the form

$$
\omega=i D q^{2}
$$

which is characteristic of a pure diffusion process. Such processes are associated with those hydrodynamic variables that do not lead to propagating modes, such as sound waves, spin waves in antiferromagnets, or transverse acoustic phonons in solids. Examples for purely diffusive processes include heat diffusion and vorticity diffusion in simple fluids and concentration diffusion in mixtures [23-25]. Neglecting cross coupling terms to other variables such diffusive modes arise from a Fourier transform of the linearized conservation law. For a concentration $c$ the latter takes the form

$$
\dot{c}+\nabla_{i} j_{i}^{c}=0
$$

with $j_{i}^{c}$, the concentration current. Assuming a linear relation between the concentration current $j_{i}^{c}$ and the thermodynamic force,

$$
j_{i}^{c}=-D^{c} \nabla_{i} \mu^{c}
$$

where $\mu_{c}$ is the chemical potential associated with the concentration $c$. Inserting eq.(3) into eq.(2), a Fourier transform of the resulting equation leads immediately to an expression of the form (1) with $D=\gamma D^{c}$, where $\gamma$ is the static susceptibility $\partial \mu^{c} / \partial c$.

Next we analyze the $T$-jump and $p$-jump experiments in the framework of a macroscopic description. We take the strain tensor $\epsilon_{i j}$ to characterize the transient positional 
order [17] - a concept well known for polymer solutions and melts [26] - and the traceless symmetric tensor $Q_{i j}[27,28]$ to describe transient orientational order [17]. For the dynamic equations for those quantities we have

$$
\begin{aligned}
\frac{\partial}{\partial t} Q_{i j}+Y_{i j} & =-\xi_{1} P_{i j} \\
\frac{\partial}{\partial t} \epsilon_{i j}+X_{i j} & =-\xi_{2} \Phi_{i j}
\end{aligned}
$$

where the relaxational character of $Q_{i j}$ and $\epsilon_{i j}$ is obvious due to the source terms on the right hand side, which contains two dissipative transport parameters $\xi_{1,2}$. In eqs.(4) and (5) we have neglected the dissipative crosscouplings $\left(\xi_{3} \Phi_{i j}\right.$ and $\xi_{3} P_{i j}$ in (4) and (5), respectively), which are generally assumed to be negligible. The conjugate quantities $P_{i j}$ and $\Phi_{i j}$ will be discussed below. $Y_{i j}$ and $X_{i j}$ are the quasi-currents associated with transient orientational order and transient positional order, respectively. They contain mainly crosscouplings to flow, which we will not consider in the following.

To further elucidate the macroscopic consequences of changes in temperature $T$ and pressure $p$ or, equivalently, in density, $\rho$ and entropy density $\sigma$, we write down the free energy density expression including $Q_{i j}$ and $\epsilon_{i j}$

$$
\begin{aligned}
f & =f_{0}+\frac{1}{2} \alpha Q_{i j} Q_{i j}+\frac{1}{2} C_{i j k l} \epsilon_{i j} \epsilon_{k l}+\left(\chi^{\rho} \delta \rho+\chi^{\sigma} \delta \sigma\right) \epsilon_{i i} \\
& +\zeta^{\sigma}\left(\nabla_{i} \sigma\right)\left(\nabla_{j} Q_{i j}\right)+\zeta^{\rho}\left(\nabla_{i} \rho\right)\left(\nabla_{j} Q_{i j}\right)
\end{aligned}
$$

where $f_{0}$ contains all the terms familiar from the hydrodynamics of simple fluids [2325]. Eq.(6) is obtained by an expansion up to second order in $Q_{i j}$ and $\epsilon_{i j}$ including all contributions allowed by symmetry; in writing down eq.(6) we have neglected a possible static cross-coupling term $U Q_{i j} \epsilon_{i j}$ between (transient) rotational and positional order.

Inspecting eq.(6), one sees immediately that there is a qualitative difference in the way the strain $\epsilon_{i j}$ and the orientational order parameter $Q_{i j}$ couple to variations of conserved variables such as density and entropy density - and therefore also to temperature $T$ and pressure $p$. While there are spatially homogeneous coupling terms to transient positional order, such terms do not exist for the orientational order parameter $Q_{i j}$ to quadratic order in the variables in the density of the free energy $f$, because $Q_{i j}$ is traceless. The contributions in the second line of eq.(6) describe coupling terms between spatial variations of transient orientational order and spatial variations of the conserved variables. We also note that an expression of the form of eq.(6) has - to our knowledge - not been presented before for the sponge phase or related isotropic phases with transient orientational and/or positional order.

As usual the thermodynamic forces are obtained from the free energy by partial differentiation. We note that the the $T$-jump and $p$-jump experiments showed a purely relaxational behavior, meaning that gradient terms are not important when describing the experimental data.

$$
\begin{aligned}
P_{i j} & \equiv \frac{\partial f}{\partial Q_{i j}}=\alpha Q_{i j} \\
\Phi_{i j} & \equiv \frac{\partial f}{\partial \epsilon_{i j}}=C_{i j k l} \epsilon_{k l}+\left(\chi^{\rho} \delta \rho+\chi^{\sigma} \delta \sigma\right) \delta_{i j}
\end{aligned}
$$


where $C_{i j k l}$ is the elastic tensor (containing 2 elastic moduli, $C_{\|}$and $C_{\perp}$ ), $\alpha$ describes the stiffness of orientational order and $\chi^{\rho}$ and $\chi^{\sigma}$ denote the coupling strength to density and entropy density. In addition we have for temperature $T$ and pressure $p$

$$
\begin{aligned}
\delta T & \equiv \frac{\partial f}{\partial \sigma}=\frac{T}{C_{V}} \delta \sigma+\frac{1}{\rho \alpha_{s}} \delta \rho+\chi^{\sigma} \epsilon_{i i} \\
\delta \mu & \equiv \frac{\partial f}{\partial \rho}=\frac{1}{\rho^{2} \kappa_{s}} \delta \rho+\frac{1}{\rho \alpha_{s}} \delta \sigma+\chi^{\rho} \epsilon_{i i}
\end{aligned}
$$

where the additional static susceptibilities contained in (9) and (10) are the specific heat (at constant density) $C_{V}$, the isentropic compressibility $\kappa_{s}$ and the adiabatic volume expansion coefficient $\alpha_{s}$.

Inspecting eqs.(8-10) we see immediately that pressure and temperature variations drive transient positional order giving rise to a transient network. After the jump, this network decays with the relaxation time $(5,8) \tau_{\epsilon} \equiv 1 /\left(\xi_{2} C_{\|}\right)$, which has to be identified with the experimental times $\tau_{P}$ and $\tau_{T}$.

Due to the static crosscoupling neglected above, also transient orientational order is generated, but only to a small amount $\left(\sim U^{2}\right)$, giving rise to a second relaxation, which was, however, not observed.

Next we turn to the discussion of the experimental result for the electric field. An electric field couples to (transient) orientational and positional order via two contributions to the free energy density

$$
f_{E}=\epsilon_{1} Q_{i j} E_{i} E_{j}+\epsilon_{2} \epsilon_{i j} E_{i} E_{j}
$$

which has to be added to (6). For the associated forces we then obtain

$$
\begin{aligned}
P_{i j} & \equiv \frac{\partial f}{\partial Q_{i j}}=\epsilon_{1} E_{i} E_{j}+\alpha Q_{i j} \\
\Phi_{i j} & \equiv \frac{\partial f}{\partial \epsilon_{i j}}=\epsilon_{2} E_{i} E_{j}+C_{i j k l} \epsilon_{k l}
\end{aligned}
$$

Thus, an external electric field in principle creates orientational as well as positional order. The latter effect, however, is usually not detectable and is therefore neglected here. The observed single relaxation time $\tau_{E}$ after removing the field can then be identified with the relaxation time of the orientational order, $\tau_{Q} \equiv 1 /\left(\xi_{1} \alpha\right)$ according to eqs. $(4,11)$.

For the associated change in the index of refraction for the case of electric birefringence we find, along the lines of [27],

$$
\Delta n=\frac{1}{2 \bar{n}}\left(\frac{\epsilon_{1}}{\alpha}+\frac{\epsilon_{2}}{C_{\|}}\right) E^{2}
$$

the experimentally observed quadratic field dependence, where the coefficient $\bar{n}$ contains the average index of refraction. Again we expect the contribution from the transient orientational order to be the important one $\left(\epsilon_{1} / \alpha \gg \epsilon_{2} / C_{\|}\right)$.

Thus we arrive at the conclusion that with $p$-jump and $T$-jump experiments one observes - as already discussed above - predominantly the effects of an induced transient network, while with $E$-jump experiments one probes mainly transient orientational order.

We therefore conclude that in the dynamic experiments described in ref. [22] one has observed both, transient positional as well as transient orientational order. This result is thus compatible with the conclusions of our analysis based on the results of early flow birefringence experiments published about a decade ago [17]. 


\section{Conclusions and perspective}

In the last section we have analyzed recent experimental results on the sponge phase and we have shown that they can be interpreted macroscopically in terms of the simultaneous presence of transient orientational and transient positional order. Naturally the question arises how this type of order for the sponge phase could be tested further experimentally. One option has already been pointed out a while ago [17], namely to investigate transverse sound waves, which would propagate above a certain frequency $\tau_{\Phi}^{-1}$ characteristic for the transient nature of the positional order in the sponge phase in the presence of transient positional order, $\omega>>\tau_{\Phi}^{-1}$, while it would still be overdamped in the low frequency regime $\omega<<\tau_{\Phi}^{-1}$. To detect this cross-over one could either use classical sound techniques or a piezo-rheometer set-up, which has turned out to be very useful to investigate the small amplitude response of various complex fluids under small shear strains including liquid crystalline elastomers [29-32] and liquid crystalline [33] as well as ordinary polymers [34]. We also note, that the three relaxation times observed in T-jump experiments by Waton and Porte [21] cannot be understood in the framework of the picture presented here. But it has been noticed before [22] that there appear to be also experimental problems in confirming this earlier result.

To further clarify and/or confirm the nature of the order parameter of the sponge phase, it would be very useful to perform more detailed measurements as the phase transitions from the sponge phase to the neighboring isotropic and lamellar phases are approached. This would be most worthwhile for materials for which these two phase transitions are only weakly first order so that one can detect significant changes in the amount of order and thus get a better handle on the modeling of these two transitions.

\section{Acknowledgements}

It is a pleasure for H.R.B. to thank P. Martinoty, H. Hoffmann and M. Gradzielski for stimulating discussions. H.R.B. also acknowledges partial support of this work by PROCOPE 312/pro-ms through the Deutscher Akademischer Austauschdienst.

\section{References}

[1] D. Roux, C. Coulon and M.E. Cates, J. Phys. Chem. 96, 4174 (1992).

[2] D.M. Anderson, H. Wennerstrom and U. Olsson, J. Phys. Chem. 93, 4243 (1989).

[3] F. Harusawa, S. Nakamura and T. Mitsui, Colloid Polym. Sci. 252, 613 (1974).

[4] J.C. Lang and R.D. Morgan, J. Chem. Phys. 73, 5849 (1980).

[5] W.J. Benton and C.A. Miller, J. Phys. Chem. 87, 4981 (1983).

[6] P.-G. Nilsson and B. Lindman, J. Phys. Chem. 88, 4764 (1984).

[7] G. Porte, J. Marignan, P. Bassereau and R. May, J. Phys. (Paris) 49, 511 (1988).

[8] G. Porte, J. Appell, P. Bassereau and J. Marignan, J. Phys. (Paris) 50, 1335 (1989).

[9] D. Roux and C. Knobler, Phys. Rev. Lett. 60, 373 (1988). 
[10] D. Gazeau, A.M. Belloq, D. Roux and T. Zemb, Europhys. Lett. 9, 447 (1989).

[11] R. Strey, W. Jahn, G. Porte and P. Bassereau, Langmuir 6, 1635 (1990).

[12] H.F. Mahjoub, C. Bourgaux, P. Sergot and M. Kleman, Phys. Rev. Lett. 81, 2076 (1998).

[13] C. Blanc and M. Kleman, Mol. Cryst. Liq. Cryst. 332, 3095 (1999).

[14] M.E. Cates, D. Roux, D. Andelman, S.T. Milner and S.A. Safran, Europhys. Lett. 5, 733 (1988).

[15] D. Roux, M.E. Cates, U. Olsson, R.C. Ball, F. Nallet and A.M. Belloq, Europhys. Lett. 11, 229 (1990).

[16] S.T. Milner, M.E. Cates and D. Roux, J. Phys. (Paris) 51, 2629 (1990).

[17] H. Pleiner and H.R. Brand, Europhys. Lett. 15, 393 (1991).

[18] G. Porte, M. Delsanti, I. Billard, M. Skouri, J. Appell, J. Marignan and F. Debeauvais, J. Phys. (Paris) II 1, 1101 (1991).

[19] C.A. Miller, M. Gradzielski, H. Hoffmann, U. Krämer and C. Thunig, Colloid Polym. Sci. 268, 1066 (1990).

[20] C.A. Miller, M. Gradzielski, H. Hoffmann, U. Krämer and C. Thunig, Prog. Colloid Polym. Sci. 84, 243 (1991).

[21] G. Waton and G. Porte, J. Phys. (France) II 4, 515 (1993).

[22] B. Schwarz, G. Mönch, G. Ilgenfritz and R. Strey, Langmuir 16, 8643 (2000).

[23] H. Pleiner and H.R. Brand, Hydrodynamics and Electrohydrodynamics of Nematic Liquid Crystals, in Pattern Formation in Liquid Crystals, eds. A. Buka and L. Kramer (Springer, New York) 1996, p. $15 \mathrm{ff}$.

[24] P.C. Martin, O. Parodi and P.S. Pershan, Phys. Rev. A 6, 2401 (1972).

[25] D. Forster, Hydrodynamic Fluctuations, Broken Symmetry and Correlation Functions (Benjamin, Reading, Mass.) 1975.

[26] H.R. Brand, H. Pleiner and W. Renz, J. Phys. France 51, 1065 (1990).

[27] P.G. de Gennes, Mol. Cryst. Liq. Cryst. 12, 193 (1971).

[28] P.G. de Gennes, The Physics of Liquid Crystals (Clarendon Press, Oxford) 1975.

[29] J.L. Gallani, L. Hilliou, Ph. Martinoty, F. Doublet and M. Mauzac, J. Phys. (France) II 6, 281 (1995).

[30] Ph. Martinoty, L. Hilliou, M. Mauzac, L. Benguigui and D. Collin, Macromolecules 32, 1746 (1999).

[31] J. Weilepp et al., Europhys. Lett. 47, 508 (1999). 
[32] J. Weilepp et al., Macromolecules 32, 4566 (1999).

[33] J.L. Gallani, L. Hilliou and Ph. Martinoty, Phys. Rev. Lett. 72, 2109 (1994).

[34] D. Collin and Ph. Martinoty, preprint, Oct. 2001. 